\title{
Temporal integration and segregation of brief visual stimuli: Patterns of correlation in time
}

\author{
VINCENT DI LOLLO \\ University of Alberta, Edmonton, Alberta, Canada \\ JOHN H. HOGBEN \\ University of Western Australia, Nedlands, Australia \\ and \\ PETER DIXON \\ University of Alberta, Edmonton, Alberta, Canada
}

\begin{abstract}
Two brief sequential displays separated by a brief interstimulus interval (ISI) are often perceived as a temporally integrated unitary configuration. The probability of temporal integration can be decreased by increasing the ISI or (counterintuitively) by increasing stimulus duration. We tested three hypotheses of the relative contributions of stimulus duration and ISI to the breakdown of temporal integration (the storage, processing, and temporal correlation hypotheses). In the first of two experiments, stimulus duration and ISI were varied factorially, and estimates of temporal integration were obtained with a form-part integration task. The second experiment was a replication of the first at two levels of stimulus intensity. The outcomes were inconsistent with the storage and processing options, but confirmed predictions from the temporal correlation hypothesis. Whether two sequential stimuli are perceived as temporally integrated or disjoint depends not on the availability of visible persistence, but on the emergence of a neural code that is based on the temporal correlation between the two visual responses.
\end{abstract}

A brief stimulus may remain visible for some time after it has been switched off. This additional period of visibility is known as visible persistence. Under a wide range of conditions, duration of visible persistence has been found to vary inversely with the duration of the inducing stimulus. For example, Efron (1970) found that, as stimulus duration was increased to $130 \mathrm{msec}$, duration of visible persistence decreased correspondingly. This relationship has come to be known as the "inverse duration effect"' (Coltheart, 1980). The effect is stable and robust, and has been obtained with a variety of experimental tasks (e.g., Allport, 1968; Bowen, Pola, \& Matin, 1974; Di Lollo, 1980; Efron, 1970; Haber \& Standing, 1969).

One consequence of the inverse duration effect was disconfirmation of the "storage"' hypothesis (e.g., Neisser, 1967). According to the storage hypothesis, visible persistence is regarded as the content of a sensory store that is charged rapidly at stimulus onset and begins discharging when the stimulus is turned off. The duration of discharge of the sensory store is held to mediate the temporal integration of brief sequential displays. For example,

This research was supported by grants from the Natural Sciences and Engineering Research Council of Canada to V.D.L. and P.D., and by a grant from the Australian Research Council to J.H. Correspondence should be addressed to V. Di Lollo, Department of Psychology, University of Alberta, Edmonton, Alberta T6G 2E9, Canada. two sequential displays, separated by a brief temporal gap (interstimulus interval, or ISI), often appear to be temporally contiguous or overlapping. According to the storage hypothesis, such temporal integration occurs if the ISI can be bridged by the decaying contents of the sensory store (i.e., by the visible persistence of the leading stimulus). Thus, two stimuli will be seen as temporally overlapping if the ISI does not exceed the period of discharge of the leading store. Assuming an initially full store and a fixed rate of discharge, temporal integration is held to depend solely on the duration of the ISI. The storage hypothesis is disconfirmed by the evidence of the inverse duration effect, because it is implausible that the duration of discharge of a store should vary inversely with the duration of charge.

Instead, the evidence is consistent with an interpretation in terms of a period of processing activity that is time locked to the onset of the inducing stimulus and indifferent to stimulus duration. According to this processing hypothesis (Di Lollo, 1980), visible persistence corresponds to a period of neural activity (which we refer to as the visual response) that starts at stimulus onset and lasts for a fixed period, irrespective of stimulus duration. The duration of visible persistence is equal to the period by which the visual response outlasts the duration of the physical stimulus. Thus, visible persistence will be longest for brief stimuli and will decrease as stimulus duration is increased 
(the inverse duration effect). Stimuli longer than the duration of the visual response are held to have no persistence at all. Several variants of this hypothesis have been proposed (Di Lollo \& Dixon, 1988; Irwin \& Yeomans, 1986; Loftus, Duncan, \& Gehrig, 1992).

Much like the storage hypothesis, the processing hypothesis postulates that temporal integration is mediated by the visible persistence of the leading stimulus (i.e., by the visual response). That is, temporal integration will occur if the duration of the leading visual response is sufficient to bridge the ISI and to overlap with the trailing visual response. Thus, for both the storage and the processing hypotheses, temporal integration depends on the degree of overlap between the two visual responses. However, the processing hypothesis differs from the storage hypothesis in one crucial aspect: The salient variable governing overlap of visual responses is not ISI but SOA (stimulus onset asynchrony: the temporal interval between the onset of the leading stimulus and the onset of the trailing stimulus).

According to the processing hypothesis, an inverse duration effect may be equivalently induced either by a longer stimulus or by a longer ISI. This is illustrated in Figure 1. The three pairs of stimuli shown in panel a of Figure 1 have the same SOA but different ISIs. According to the processing hypothesis, temporal integration should be the same in each case, as illustrated in panel $b$. This follows from the assumptions that the visual response is time locked to stimulus onset, that it has a fixed duration, and that integration is based on the degree of overlap between the two visual responses. Thus, provided that SOA remains constant, any combination of stimulus du-

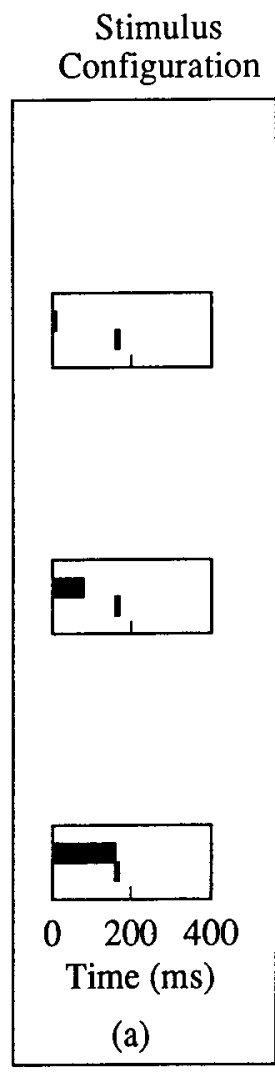
Processing Hypothesis Visual Response

Correlational Model
Visual Response

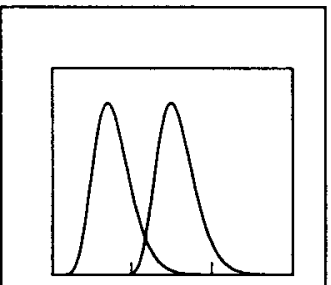

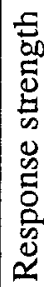

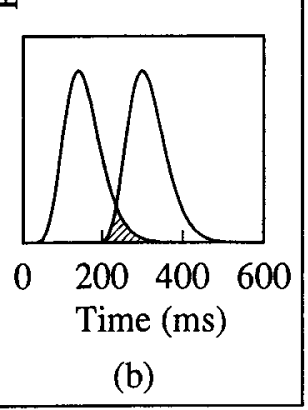

Correlational Model Response Scattergrams

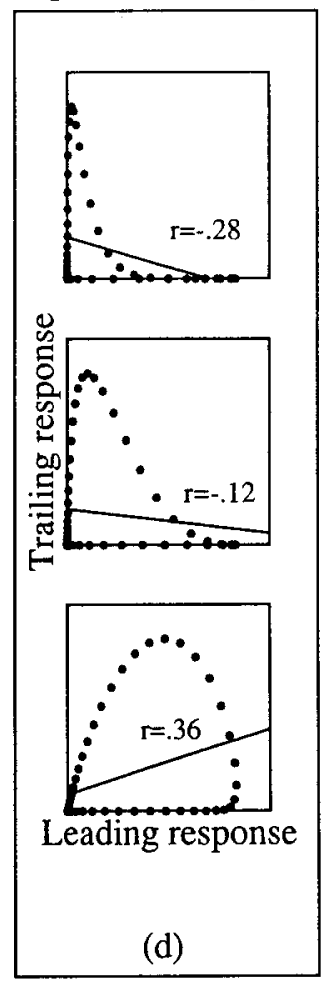

Figure 1. Temporal integration of two sequential stimuli at fixed stimulus onset asynchrony (SOA) but at varying durations of leading stimulus and interstimulus interval (ISI). The sketches are not intended to illustrate the conditions of Experiment 1; rather, they illustrate the hypothetical relationship between physical stimuli and visual responses as predicted by the processing hypothesis and by the temporal correlation model. Panel a contains timing diagrams that indicate the physical configuration of the stimuli; the ISI is longest in the stimulus pair at the top of the panel, shorter in the middle pair, and equal to zero in the lowest pair. The duration of the leading stimulus increases correspondingly. Panel b shows the visual responses generated by the corresponding stimuli according to the processing hypothesis. The predicted degree of temporal integration is indexed by the overlap between pairs of visual responses, indicated by the shaded areas. Integration is said to depend on SOA, so equal degrees of integration are predicted for each combination of stimulus duration and ISI. Panel $\mathrm{c}$ shows the visual responses generated according to the temporal correlation model. Panel d contains scatterplots of the relationship between the corresponding visual responses illustrated in panel $c$. The visual response curves in panels $c$ and $d$ represent the outputs of linear filters with input provided by the corresponding stimuli in panel a. Details of the filtering operation are specified in Equation 11 and in the related text. 
ration and ISI should produce the same level of temporal integration. In other words, stimulus duration and ISI are equivalent determinants of temporal integration.

A different prediction is made by the temporal correlation model, proposed recently by Dixon and Di Lollo (in press). According to this model, temporal integration depends not on the duration of the visible persistence of the leading stimulus, but on the correlation between the visual responses triggered by the two stimuli over time. A detailed description of the model is presented later in the text; here, we outline its basic tenets. Upon presentation, a stimulus is assumed to trigger a temporally extended visual response that can be represented by the output of a linear filter. The stimuli in panel a of Figure 1 represent the inputs to the filter; the corresponding response functions in panel $\mathrm{c}$ represent the filtered outputs. It is assumed that a correlational mechanism within the visual system takes regular samples of the two response functions and computes a correlation between the two sets of activity estimates, having first weighted them in terms of an exponentially shaped sliding temporal window. The scatterplots in panel d of Figure 1 illustrate the results of sampling and correlating the visual responses over time. The correlation coefficient associated with each scatterplot is an index of the degree of temporal integration predicted by the model.

Clearly, stimulus duration and ISI affect temporal integration differently in the processing hypothesis and in the correlational model. According to the processing hypothesis, stimulus duration and ISI are equivalent; increasing the duration of one while decreasing the other by the same amount should have no effect on temporal integration. By contrast, according to the correlational model, temporal integration should be affected more by a change in ISI than by a corresponding change in stimulus duration. These contrasting predictions were tested by studying temporal integration as a joint function of stimulus duration and duration of ISI over a range of SOAs. The outcome was as predicted by the temporal correlation model.

\section{GENERAL METHOD}

The experimental task required temporal integration of two stimuli displayed in rapid succession. Viewed separately, the stimuli appeared as random aggregates of 12 small patches of light, but, viewed together, the two stimuli portrayed 24 of the 25 elements of a $5 \times 5$ square matrix. The task of the observer was to identify the location of the missing element within the matrix. Often used for studying temporal integration (Breitmeyer, Kropfl, \& Julesz, 1982; Hogben \& Di Lollo, 1974; Shioiri \& Cavanagh, 1992), the matrix task is performed easily by most observers after little practice. At brief SOAs, the missing element stands out clearly against the integrated matrix; however, at long SOAs, the observer sees a matrix riddled with empty locations, all of which, on analysis, turn out to have been occupied by elements of the first stimulus.

\section{Observers}

Two of the authors and a colleague served as observers. All had normal or corrected-to-normal acuity.

\section{Visual Displays}

Stimuli were displayed on a Hewlett-Packard 1333 oscilloscope with $\mathrm{P} 15$ phosphor. The $x, y$, and $z$ (intensity) coordinates of points to be displayed were stored in a fast-plotting buffer (Finley, 1985) that drove the oscilloscope at a rate of 1,000 points $/ \mathrm{msec}$. At the viewing distance of $57 \mathrm{~cm}$, set by a headrest, the $8 \times 8 \mathrm{~cm}$ display surface subtended a visual angle of $8^{\circ}$ on the side. Ambient light provided screen illumination of approximately $0.1 \mathrm{~cd} / \mathrm{m}^{2}$.

The display consisted of 24 of the 25 elements defining a $5 \times 5$ matrix. Each element was made up of 36 dots that filled a small square whose side subtended $6^{\prime}$ of visual angle. Separation between elements was $36^{\prime}$ between adjacent sides. The visual angle subtended by the entire matrix was $2^{\circ} 54^{\prime}$. Four dim fixation points were displayed at the corners of a $4^{\circ}$ square area within which the matrix was centered. The observers sat in a dimly lit room and viewed the displays with natural pupils. Upon a buttonpress, the 24 matrix elements were displayed in two images of 12 elements each, separated by an ISI. The 12 elements in each image were chosen randomly on each trial from the set of 25 , so that the location of the missing element varied randomly on each trial. The observers identified the missing element by row and column, guessing if unsure, and encoded the response by using an array of buttons providing input to the computer.

\section{EXPERIMENT 1}

\section{Method}

Experiment 1 was designed to study temporal integration over a range of exposure durations of the leading stimulus and a range of ISIs. There were seven exposure durations of the first image (20, $40,60,80,100,120$, and $140 \mathrm{msec}$ ), and eight durations of ISI $(0,20,40,60,80,100,120$, and $140 \mathrm{msec})$, combined factorially for a total of 56 conditions. The duration of the second image was always $10 \mathrm{msec}$. Luminance of the displays was measured in candelas-microsecond ( $\mathrm{cd}-\mu \mathrm{sec}$ ). These are units of luminous directional energy per point that provide an appropriate description of the luminous intensity of oscilloscopic displays (Sperling, 1971). The luminance of stimuli of different durations was adjusted by the method described by Di Lollo and Finley (1986) so that all stimuli, regardless of duration, were seen to have the same brightness as the 10 -msec trailing display whose luminance was set at $0.0082 \mathrm{~cd}-\mu \mathrm{sec}$.

One experimental session comprised 20 trials at each of the eight ISIs, at one of the seven durations of the first image. The 160 trials were sequenced randomly within each session. Each observer completed a total of 35 sessions, yielding 100 trials per condition.

\section{Results and Discussion}

The results are illustrated in Figure 2, separately for each observer, as a function of SOA. Although suitable for highlighting predictions from the processing hypothesis, the pattern of curves in Figure 2 does not reveal an inverse duration effect at first glance. Clear evidence of an inverse duration effect is seen in Figure 3, where accuracy of performance is plotted as a function of stimulus duration at a fixed ISI of $40 \mathrm{msec}$. Curves similar to those in Figure 3 were obtained for other ISIs.

\section{The Processing and the Storage Hypotheses}

It is immediately apparent from Figure 2 that performance was not determined by SOA alone. Had SOA been the sole determinant, there would have been a single level 


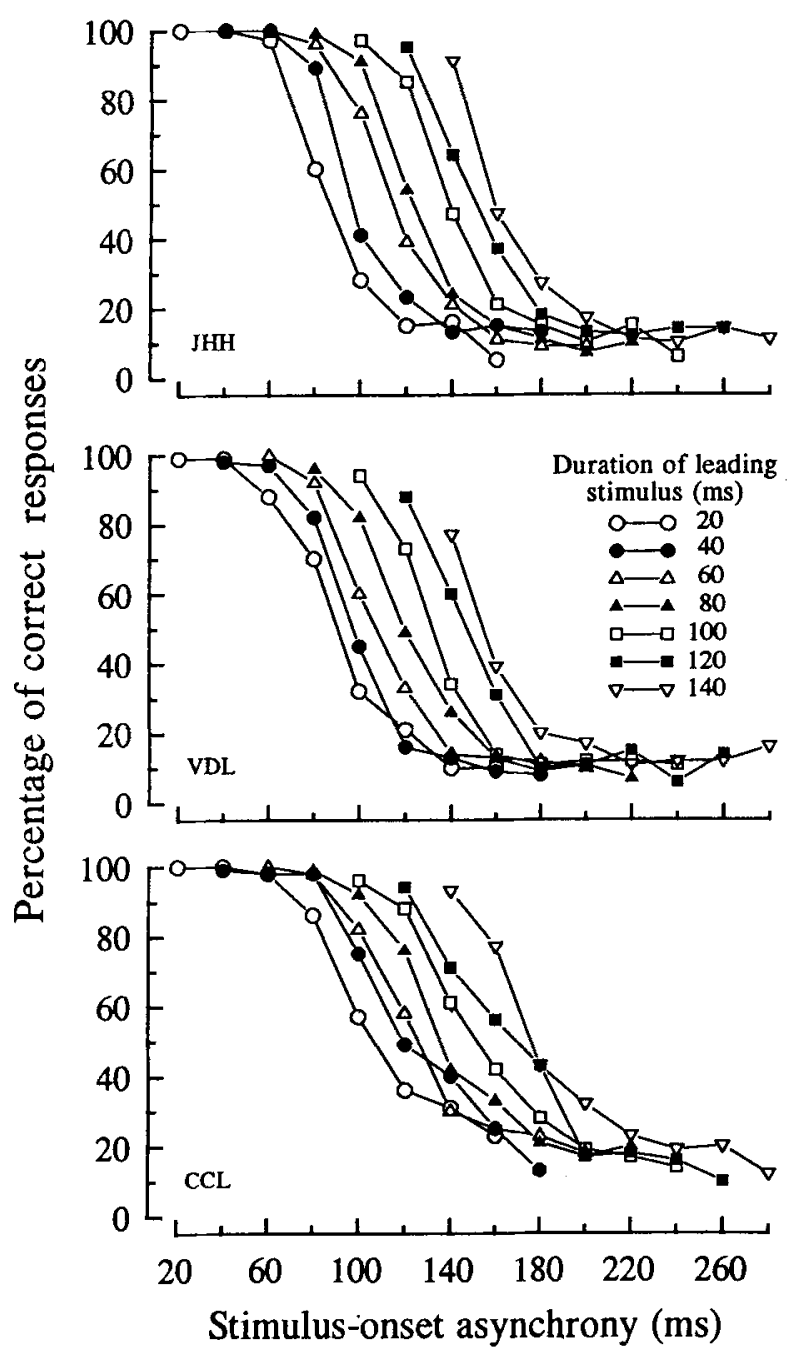

Figure 2. Performance in Experiment 1 as a function of SOA, shown separately for each observer. Separate curves are plotted for each duration of the leading display.

of performance associated with each SOA or, equivalently, a single performance curve for each observer. Instead, any given level of SOA in Figure 2 exhibits a range of performance levels, depending on the combination of exposure duration and ISI. For example, for Observer J.H.H., an SOA of $100 \mathrm{msec}$ yielded performance levels ranging between $97 \%$ (exposure duration $=100 \mathrm{msec}$, ISI $=0 \mathrm{msec}$ ) and $28 \%$ (exposure duration $=20 \mathrm{msec}$, ISI $=80 \mathrm{msec}$ ).

This pattern of results is not consistent with expectations based on the processing hypothesis. In terms of that hypothesis, temporal integration between successive stimuli should vary with the degree of temporal overlap between the visual responses initiated by the two stimuli, as shown in panel $b$ of Figure 1. In turn, degree of overlap should depend entirely on the temporal separation between the onsets of the two stimuli, that is, on the SOA. The finding that a range of performance levels is associated with a single SOA indicates that stimulus duration and/or ISI must affect performance independently of SOA.

The results are just as unsupportive of the storage hypothesis. According to that hypothesis, the sensory store is charged rapidly upon stimulus onset, continues to be charged while the stimulus is on display, and begins to discharge upon stimulus termination. Temporal integration across an ISI is said to be possible if the trailing stimulus is displayed before the leading store has had time to discharge. With respect to Experiment 1, the predicted relationship between stimulus duration and accuracy of performance is unambiguous: Performance might conceivably improve as stimulus duration (i.e., duration of charge) is increased. However, given a full store, performance should be unaffected by exposure duration. Notably, under no circumstances should an increment in exposure duration lead to a decrement in performance. To the contrary, Figure 3 shows striking decrements in performance as stimulus duration is increased with ISI held constant.

An aspect of the results not immediately apparent in Figures 2 and 3 can be seen in Figure 4, which shows contour plots of accuracy as a function of stimulus duration and ISI. The transitions between gray bands in Figure 4 represent iso-accuracy contours for $25 \%, 50 \%$, and $75 \%$ correct responses. The heavy contour lines represent predictions from the correlational model described below; they should be ignored for the moment.

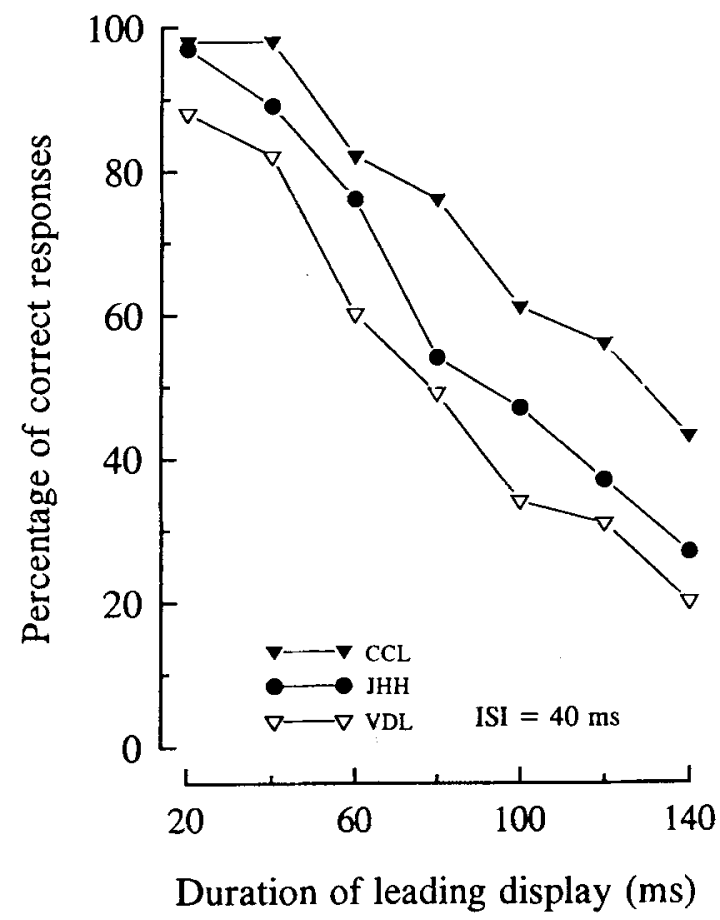

Figure 3. Performance in Experiment 1 as a function of duration of the leading display at an ISI of $\mathbf{4 0} \mathrm{msec}$. A strong inverse duration effect is revealed by the negative slope of the performance curve of each observer. 

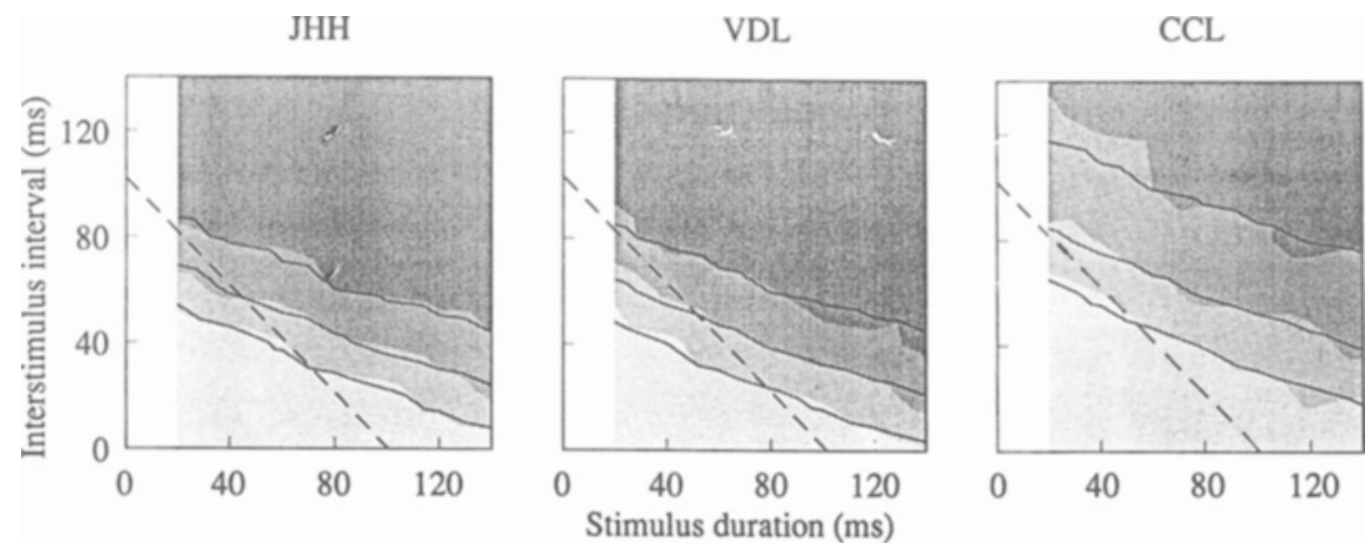

Figure 4. Contour plots of accuracy of performance in Experiment 1 as a function of stimulus duration and ISI. From darkest to lightest, the shaded areas represent the following ranges of correct responses: $0 \%-25 \%, 25 \%-50 \%$, $50 \%-75 \%, 75 \%-100 \%$. Thus, the transitions between gray bands represent iso-accuracy contours for $25 \%, 50 \%$, and $75 \%$ correct responses. The heavy contour lines represent predictions from the temporal correlation model for proportions of $.25, .50$, and .75 correct responses. The segmented lines in each panel illustrate predictions from the processing hypothesis, as explained in the text.

For each observer, the iso-accuracy contours are slanted downward along the duration axis. The slant of these contours is of interest because it conflicts with predictions from both the storage and the processing hypotheses. According to the storage hypothesis, the iso-accuracy contours should be horizontal and flat throughout the domain. This is because, given a full sensory store, temporal integration (and, therefore, accuracy of performance) is held to be determined solely by ISI. Thus, for any given ISI, changing the duration of the stimulus should have no effect on performance; this leads to the following predictions. First, the overall level of each iso-accuracy contour should be determined entirely by the ISI (the longer the ISI, the higher the contour's overall level in Figure 4). Second, each contour should remain horizontal throughout the domain, because stimulus duration is held to have no effect on performance. Contrary to this prediction, the iso-accuracy contours in Figure 4 exhibit uniformly downward slants, indicating that stimulus duration affected performance even if ISI was held constant.

Predictions from the processing hypothesis are just as straightforward. As illustrated in Figure 1, the processing hypothesis holds that temporal integration is determined entirely by SOA, namely, by the sum of ISI and stimulus duration. Thus, given a fixed SOA, level of performance should remain the same regardless of what combination of stimulus duration and ISI makes up the SOA. In fact, by varying stimulus duration and ISI systematically while leaving the SOA fixed, it is possible to construct iso-SOA contours in Figure 4. One such contour (for an SOA of $100 \mathrm{msec}$ ) is shown by the segmented line in each panel of Figure 4. The slant of the segmented lines is constrained by the consideration that, for SOA to remain invariant as stimulus duration is increased, ISI must be decreased by the same amount. A moment's reflection will show that, because accuracy is held to depend solely on SOA, iso-accuracy contours should coincide with iso-SOA contours. Notably, both sets of iso-contours should have equivalent slant. But this is patently not the case: the iso-accuracy contours in Figure 4 have a slant that is far shallower than that of the iso-SOA contours represented by the segmented lines. This indicates that, contrary to the processing hypothesis, stimulus duration and ISI are not equivalent determinants of temporal integration. In fact, temporal integration is hindered far more by an increment in ISI than by a corresponding increment in stimulus duration.

\section{The Temporal Correlation Model}

A new account of temporal integration has been proposed by Dixon and Di Lollo (in press). Its principal tenet is that temporal integration depends not on the availability of visible persistence, but on the emergence of a neural code that determines whether sequential stimuli will be perceived as temporally integrated or as temporally disjoint. The model is couched on the premise that, in processing trains of stimuli, the visual system must accommodate two competing requirements: to construct detailed representations of objects and scenes, and to detect rapid changes in the visual environment. To maximize the accumulation of detailed information, a suitable perceptual strategy is to integrate incoming stimuli over a period of time. In doing so, however, temporal resolution within the period of integration is lost. On the other hand, if sudden changes in the environment are to be detected rapidly, the opposite strategy is required: sequential stimuli should be perceived as temporally separate or disjoint. The rules governing temporal integration and segregation do not seem to be based on a simple principle such as duration of the ISI. Indeed, it is possible for stimuli separated by a sizable ISI to be perceived as temporally integrated, and for stimuli that actually overlap in time to be perceived 
as temporally disjoint. The correlational model describes one way in which temporal integration and segregation of sequential visual stimuli may be achieved.

We have proposed (Dixon \& Di Lollo, in press) that perception of the temporal relationship between two stimuli is determined by the outcome of a sensory coding process that correlates the visual responses over time. If the correlation is high (i.e., if the visual responses form similar patterns over time), the stimuli are coded as temporally coextensive and are perceived as belonging to the same object or event. On the other hand, if the correlation is low, the stimuli are coded as temporally disjoint and are perceived as being independent of each other.

This approach bears notable similarities to correlational models of spatial vision (e.g., Dodwell, 1971; Glass, 1969; Uttal, 1975). In those models, correlational principles are used to index the similarity of spatial patterns; in the present model, the same principles are used to index the similarity of temporal patterns. Application of the temporal coding model to the outcome of Experiment 1 requires the three procedural steps described below: filtering the stimuli, sampling and correlating the visual responses, and predicting performance.

Filtering the stimuli. We make the axiomatic assumption that temporal integration and segregation arise from interactions not between the physical stimuli, but between the corresponding visual responses. The transfer from physical stimuli to visual responses is predicated on the assumption that, in its peripheral stages, the visual system acts as a linear temporal filter (Ives, 1922; Kelly, 1961). On this assumption, any given visual response can be represented as the output of a linear filter with input provided by the physical stimulus.

The functions illustrated in panel $c$ of Figure 1 represent outputs of the filtering operation. In each case, the filter's inputs are provided by the physical stimuli whose luminance profiles are shown in panel a. By assumption, each function in panel $\mathrm{c}$ represents a visual response, namely, the spurt of activity produced within the visual system by the corresponding stimulus in panel a.

Sampling and correlating the visual responses. The visual code that determines whether two stimuli will be perceived as temporally coextensive or disjoint is based on the correlation between the two visual responses. To arrive at an estimate of the correlation for any given configuration of stimulus duration and ISI, we proceed as follows.

1. We assume that the visual responses are sampled continually over time so as to yield a series of activity estimates on which the correlation is computed. Illustrative sets of such activity estimates for three pairs of visual responses are shown by the scatterplots in Figure 1, panel $\mathrm{d}$. Elements in a scatterplot represent separate estimates of the activity levels of the two response functions at 10 -msec intervals.

2. Activity estimates are assumed to decay over time and, therefore, to contribute in different measures to the correlation. New estimates are given the highest weights, whereas earlier estimates are given weights that diminish progressively as a function of the rate of temporal decay. In essence, the rate of decay defines a sliding temporal window that is akin to Allport's (1968) concept of traveling moment. This refers to a brief temporal period (of the order of $100 \mathrm{msec}$ ) that contains a running sample of the visual input; new stimuli enter the moment as it travels forward in time, and old stimuli become progressively weaker and eventually disappear.

3. Each time a new activity estimate is obtained, new weights are assigned to all earlier estimates in accordance to the rate of temporal decay, and a new correlation is calculated. This procedure ensures that new estimates of the correlation continue to be produced at a rate that matches that of the sampling process.

4. The activity estimates represented by the individual points in the scattergrams in panel d of Figure 1 were calculated for idealized conditions. That is, the visual responses were assumed to be noise free, their time courses were assumed to remain unchanged from trial to trial, and the sampling mechanism was assumed to be perfectly timed. It goes without saying that, in a biological system, none of these mechanisms would be noise free. This is tantamount to saying that the estimate of the correlation for any given combination of stimulus duration and ISI should be expected to vary from trial to trial. The degree of variation will depend on both the levels and the sources of noise within the system.

At a first approximation, we assume that the activity samples (on which individual estimates of the correlation are based) are drawn from a bivariate normal distribution with correlation $\rho$. In turn, the noisy estimates of the correlation will form a probability distribution with mean $\rho$, as shown in Figure 5. It is understood that $\rho$ will vary for different combinations of stimulus duration and ISI (see Figure 1).

The distribution illustrated in Figure 5 provides the basis for deciding whether two stimuli will be perceived as temporally coextensive or disjoint. Specifically, two stimuli will be perceived as coextensive (i.e., as temporally integrated) when the estimated correlation $r$ is greater than some criterion $r_{c}$. In order to calculate the probability of integration, we use the well-known $z^{\prime}$ transformation of the product-moment correlation developed by Fisher (1922). According to this approximation, the probability of temporal integration $\left(P_{i}\right)$ is given by

$$
P_{i}=P\left(r>r_{c}\right)=P\left(z>z_{c}\right),
$$

where

$$
z_{c}=\frac{1}{2} \ln \left(\frac{1+r_{c}}{1-r_{c}}\right)
$$

and $z$ is approximately normally distributed with mean

$$
z_{\rho}=\frac{1}{2} \ln \left(\frac{1+\rho}{1-\rho}\right)
$$

and variance $(n-3)^{-1}$, where $n$ is the effective sample 


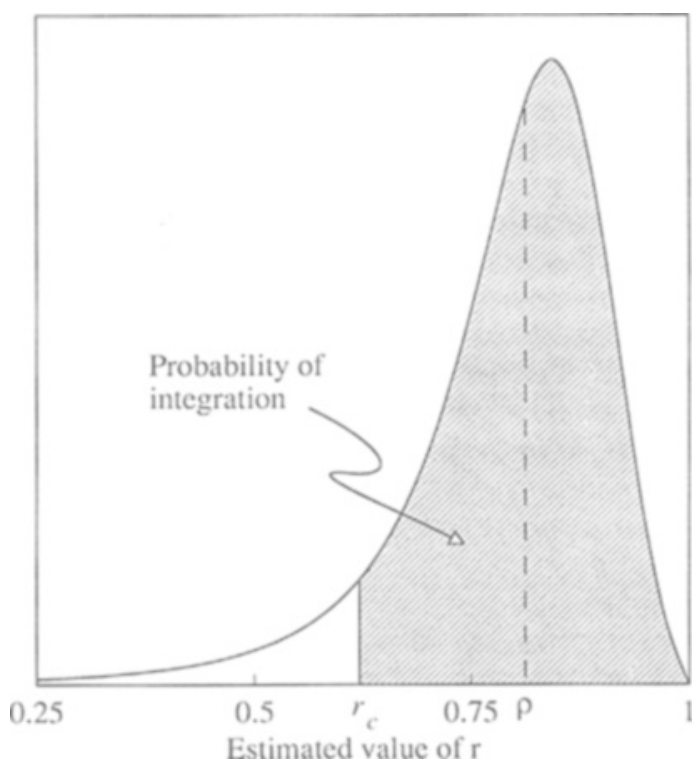

Figure 5. Sampling distribution of the correlation between visual responses according to the temporal correlation model. The mean of the distribution $(\rho)$ is determined jointly by stimulus duration and ISI. The criterial correlation $\left(r_{c}\right)$ separates integration from segregation areas in the sampling distribution. If the correlation between two visual responses exceeds $r_{c}$ (shaded area), the corresponding stimuli will be perceived as temporally integrated; if not, the stimuli will be seen as temporally disjoint.

size. The effective sample size is determined jointly by the rate of sampling of the visual responses and by the rate of decay of the activity estimates. With some algebraic manipulation, Equation 1 can be written as

$$
P_{i}=P\left[z^{\prime}>(n-3)\left(z_{c}-z_{\rho}\right)\right],
$$

where $z^{\prime}$ is approximately normally distributed with zero mean and unit variance. Further, the normal distribution can be approximated with a logistic distribution

$$
F_{(z)}=\frac{e^{2 z}}{1+e^{2 z}} \text {. }
$$

Using this approximation, Equation 4 can be rewritten as

$$
\begin{aligned}
P_{i} & =\frac{\exp \left[2(n-3)\left(z_{p}-z_{c}\right)\right]}{1+\exp \left[2(n-3)\left(z_{\rho}-z_{c}\right)\right]} \\
& =\frac{\left(\rho^{\prime}\right)^{n-3}}{\left(\rho^{\prime}\right)^{n-3}+\left(r_{c}^{\prime}\right)^{n-3}},
\end{aligned}
$$

where

$$
\rho^{\prime}=\frac{1+\rho}{1-\rho}
$$

and

$$
r_{c}^{\prime}=\frac{1+r_{c}}{1-r_{c}} .
$$

Predicting performance. Finally, we present a procedure for computing the probability of a correct response in Experiment 1 as a function of the correlation between visual responses. First, we note that when two stimuli are perceived as coextensive and are temporally integrated, performance in the dot matrix task is generally accurate. However, accuracy may not be perfect; occasional errors may be caused by stray eye movements, lapses of attention, or by coding a response incorrectly. We use the parameter $a$ to indicate the peak level attained when the stimuli are fully integrated. Under most circumstances, $a$ will be close to unity.

Next, we consider what happens when the two parts of the matrix are not integrated. In this case, observers have no clear sense of the location of the missing dot, and a response must be made by guessing. However, observers do not guess randomly; we know that most errors consist of selecting a location that contained a dot in the leading stimulus (Di Lollo, 1980). That is, observers can remember the locations of the dots in the trailing image and avoid them when making a response. In addition, observers may remember the locations of some dots in the leading display, and also avoid selecting those as responses. We use the parameter $g$ to denote the level of accuracy attained by using guessing strategies of this sort.

On these assumptions, the probability of a correct response $\left(P_{c}\right)$ can be written as

$$
P_{c}=a P_{i}+g\left(1-P_{i}\right)=g+(a-g) P_{i} .
$$

Combining Equations 6 and 9 yields an expression for the predicted probability of correct responses as a function of $\rho$ (which depends jointly on stimulus duration and ISI) and the parameters of the model

$$
P_{c}=g+(a-g) \frac{\left(\rho^{\prime}\right)^{n-3}}{\left(\rho^{\prime}\right)^{n-3}+\left(r_{c}^{\prime}\right)^{n-3}} .
$$

Fitting the model to the data required five parameters, which were estimated by using a gradient descent search that minimized the squared error of prediction. In estimating $\tau$, the temporal constant of the linear filter, we followed earlier practice (Dixon \& Di Lollo, in press) and assumed that the impulse response was described by the gamma function

$$
I(t)=\frac{t^{m-1} e^{t / \tau}}{\tau^{m} \Gamma(m)} .
$$

We also followed earlier practice in setting the value of $m$ at 10 (see also Watson, 1986). Next, we computed the effective number of activity estimates to be used in calculating the correlation ( $n$ in Equations 6 and 10). The decay of these activity estimates was assumed to be exponential with a time constant of $340 \mathrm{msec}$, a value estimated from the data reported by Dixon and Di Lollo (in press). The third parameter was $r_{c}$, the criterial correlation given in Equation 1. The final two parameters to be estimated from the data were $a$ and $g$ (Equations 9 and 10 ), which determine the probability of a correct response given that the stimuli are integrated or segregated, respectively.

Predictions based on Equation 10 are illustrated in Figure 6. Within each panel, the continuous curve shows the 
model's fit of an observer's performance in Experiment 1. Individual dots in Figure 6 represent the proportion of correct responses for each observer in each condition of Experiment 1. Details of the parametric fit are given in Table 1.

It is manifest from the fits in Figure 6 that the temporal correlation model provides an excellent account of the results of Experiment 1. The success of the model relative to the storage and the processing hypotheses can be assessed by referring to Figure 4 . The heavy contour lines in Figure 4 represent the model's estimates for proportions of $.25, .50$, and .75 correct responses. The estimated levels are remarkably close to the empirical levels for each observer. It is especially noteworthy that the slants of the contour lines closely match the slants of the gray bands. This is a far closer match than that provided by either the processing or the storage hypotheses which, as was noted above, predict steeper or shallower slants, respectively.

A question of generality must now be considered. Undoubtedly, the temporal correlation model can account for the relative effects of stimulus duration and ISI. Can the same correlational principles provide credible accounts of other known effects in temporal integration? Besides the inverse duration effect studied in Experiment 1, at least one other variable-stimulus intensity-is known to affect temporal integration of brief sequential stimuli. In Experiment 2, we investigated whether the same temporal correlational principles that can account for the inverse
Table 1

Separate Parameter Estimates for Each Observer in Experiment 1

\begin{tabular}{crrr}
\hline Parameter & J.H.H. & V.D.L. & C.C.L. \\
\hline$\tau^{*}$ & 16.8 & 15.5 & 18.1 \\
$n$ & 6.95 & 5.99 & 6.22 \\
$r_{c}$ & 0.440 & 0.442 & 0.362 \\
$a$ & 1.000 & 1.000 & 0.982 \\
$g$ & 0.094 & 0.048 & 0.129 \\
Root-mean squared & 0.031 & 0.040 & 0.040 \\
error of prediction & & & \\
\hline
\end{tabular}

*In milliseconds.

duration effect can provide a consistent account of the effect of intensity of stimulation.

\section{EXPERIMENT 2}

Duration of visible persistence is known to be related inversely to the intensity of stimulation. This inverse intensity effect (Coltheart, 1980) can be obtained in either of two ways: by varying the intensity of the stimuli on a background of fixed luminance (Allport, 1968; Efron \& Lee, 1971; Hogben \& Di Lollo, 1985) or by varying the intensity of the background on which the stimuli are presented (Dixon \& Hammond, 1972). In either case, the duration of visible persistence diminishes as the intensity of stimulation is increased. In Experiment 2, we aimed at obtaining an inverse intensity effect by replicating Ex-

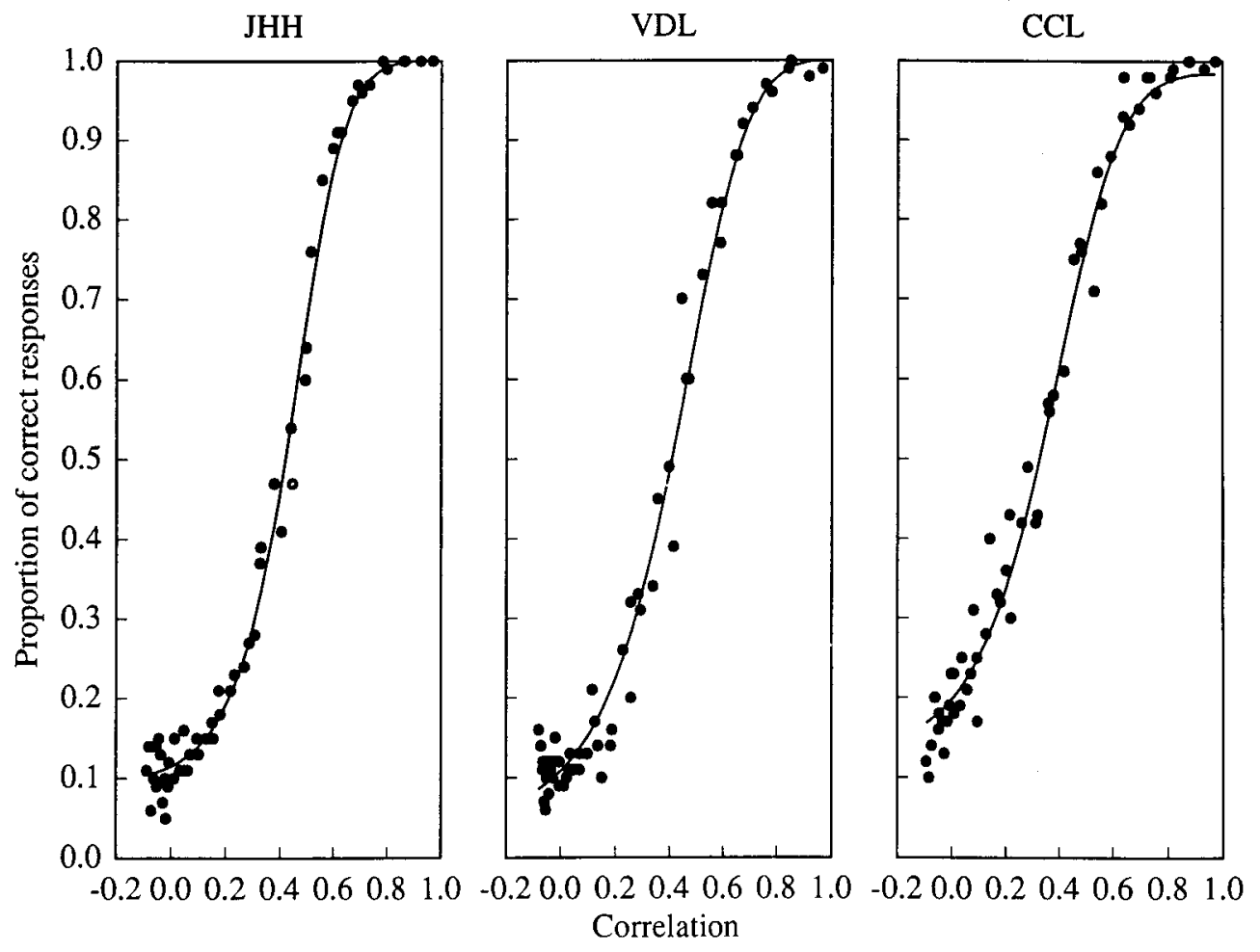

Figure 6. Relationship between accuracy of performance in Experiment 1 and the correlation between visual responses computed on the basis of Equation 10. Within each panel, the continuous curve shows the model's fit to an observer's performance. Individual dots represent the proportion of correct responses obtained by each observer in each condition of Experiment 1. Details of the parametric fit are given in Table 1. 
periment 1 at two levels of stimulus intensity. The major purpose of the study was to check whether the results could be encompassed within the temporal correlation model.

In deciding what construct might best handle the effect of intensity within the model, a plausible working hypothesis can be based on the temporal constant of the input filter. If the temporal constant, and hence the duration of the visual response, were to vary inversely with the intensity of stimulation, the inverse intensity effect would follow. To wit, longer visual responses for dimmer stimuli would lead to higher temporal correlations and thus to higher probabilities that the two parts of the matrix would be coded as coextensive.

This working hypothesis derives a good deal of plausibility from similar work done on the duration of the critical period of temporal summation, known as Bloch's law, at threshold. The duration of the critical period is known to vary inversely with the intensity of stimulation. As in the case of the inverse intensity effect, the critical period diminishes with increments in the intensity of either the adapting background (Roufs, 1972a) or the stimuli (Hood \& Grover, 1974; Ueno, 1977). A formal model has been developed to account for the effect of varying background intensity (Roufs, 1972b), though not for varying intensity of single pulses presented on a background of fixed luminance. In that model, the temporal constant of the impulse response function becomes smaller as background intensity is increased. In turn, this reduces the period during which temporal summation can occur. There is little question that-mutatis mutandis-this reasoning can be applied to a temporal correlation account of the inverse intensity effect obtained with changes in background level. Our working hypothesis is that a similar formal model (perhaps along the lines suggested recently by Graham \& Hood, 1992) might apply to single pulses on constant backgrounds. In fact, the outcome of the experiment was in agreement with such an hypothesis.

\section{Method}

Experiment 2 was a replication of Experiment 1 at two levels of stimulus intensity. In the dim condition, the brightness of the stimuli of all durations matched that of the $10-\mathrm{msec}$ trailing display whose luminance was set at $0.0055 \mathrm{~cd}-\mu \mathrm{sec}$. Similarly, in the bright condition, all stimuli were brightness-matched to the 10 -msec trailing display whose luminance was set at $0.0866 \mathrm{~cd}-\mu \mathrm{sec}$. The former yielded stimuli that were dimly but clearly visible; the latter yielded bright stimuli that were almost flaring. The only other change was the addition of a 10-msec duration of the leading display. This was purely an exploratory measure intended to extend the range of very brief SOAs.

\section{Results and Discussion}

The results are shown in Figure 7. For clarity of presentation, the results for each observer have been split between two graphs (see caption of Figure 7). The results are orderly and consistent with the corresponding results of Experiment 1 (Figure 2). A negative intensity effect is indicated by a uniform shift of the curves for the dim condition toward the longer ISIs. However, the regularity of the shift is not obvious at first sight.

A clearer cross section of the results is provided in Figure 8 , which shows the critical ISI (obtained by linear interpolation between the two data points straddling the $50 \%$ level) that yielded $50 \%$ correct responses at each duration of the leading stimulus in both dim and bright viewing conditions, separately for each observer. Also illustrated in Figure 8, for comparison, are the corresponding critical ISIs (50\% correct responses) obtained in Experiment 1 . Both an inverse duration and an inverse intensity effect can be seen in Figure 8 . An inverse duration effect is revealed by the systematic decrement in critical ISI across the domain: as stimulus duration is increased, the extent of temporal integration-as indexed by the critical ISI-decreases apace. An inverse intensity effect is evidenced by the longer critical ISIs (by an average of $10 \mathrm{msec}$ ) in the dim than in the bright condition. By inference, the extent of temporal integration is longer with dim than with bright stimuli. As shown by the evidence in Figure 8, the inverse intensity effect was asymptotic for 2 of the 3 observers at the stimulus luminance employed in Experiment 1.

The temporal correlation model described in Experiment 1 was applied to the data of Experiment 2. Parameters for the dim and the bright conditions were estimated simultaneously, with the constraint that all parameters be the same in the two conditions except for the temporal constant of the input filter. This was done in accordance with the hypothesized relationship between luminance level and the temporal extent of the visual response function. The parametric fits listed in Table 2 show that, as expected, the temporal constant of the input filter was longer in the dim than in the bright condition for each observer. As a further test, the model was fit to the data of Experiments 1 and 2 simultaneously, using separate values of $\tau$ for Experiment 1 and for the bright- and the dim-dot conditions of Experiment 2. The model fits the combined experiments as well as it does in either experiment alone. The estimated parameters and errors of prediction are shown in Table 3.

Figure 9 shows the model's fit to the combined data for both luminance conditions in Experiment 2. As was the case in Experiment 1 (Figure 6), the fit is remarkably accurate (see Table 2 ). A more comprehensive overview of the results is given in Figures 10 and 11, where the data are presented as a function of stimulus duration and ISI separately for the dim and the bright viewing conditions. The heavy contour lines represent predictions from the temporal correlation model. As in Experiment 1, the model provides an excellent fit to the contour plots.

Two related aspects of the success of the temporal correlation model in predicting the outcome of Experiment 2 should be noted. First, as had been surmised, the inverse intensity effect is handled successfully within the model by appropriate modulation of the temporal constant of the 


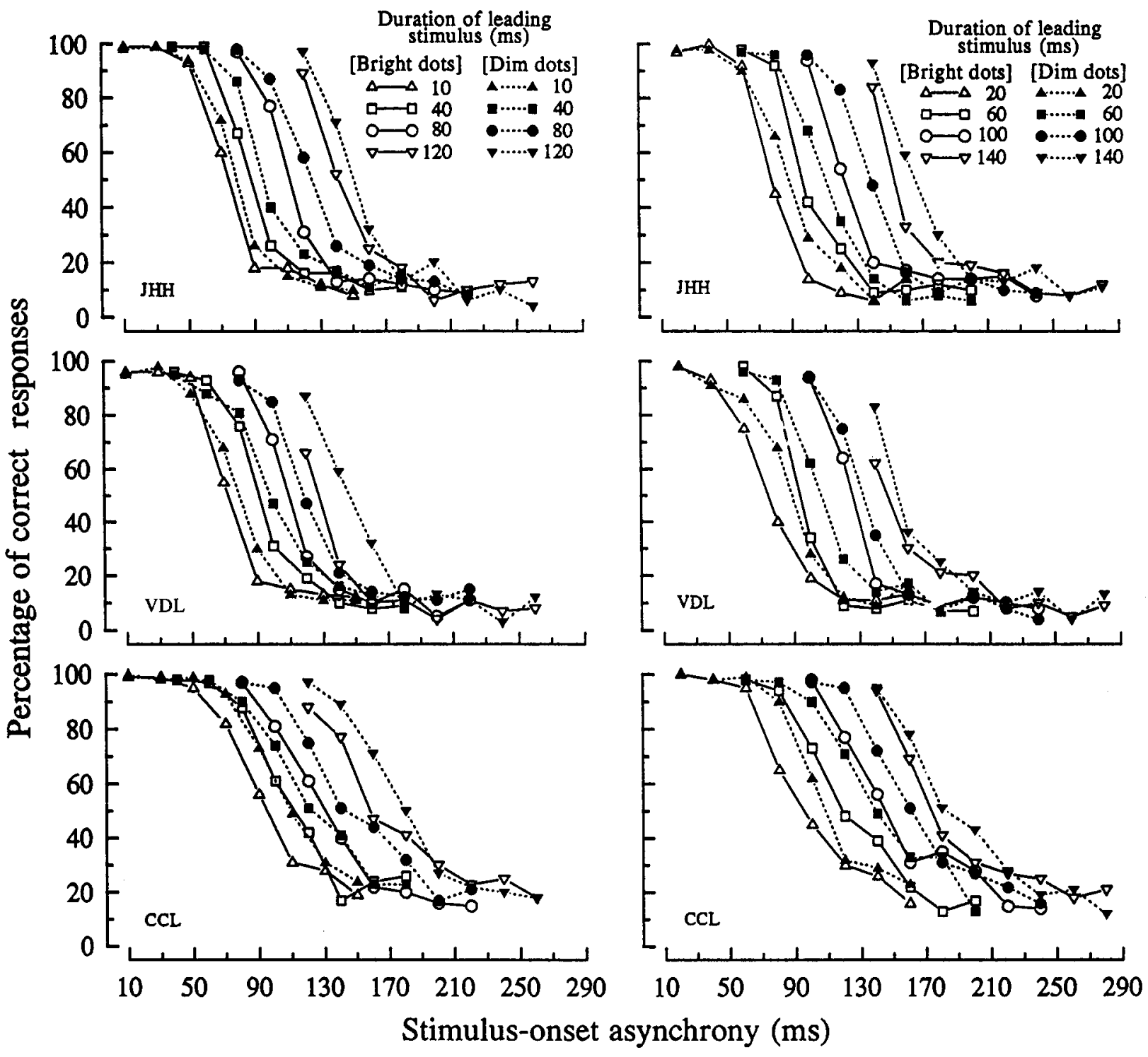

Figure 7. Performance in Experiment 2 as a function of stimulus onset asynchrony, shown separately for each observer. Separate curves are plotted for each duration of the leading display in the dim and the bright viewing conditions. If the data are plotted in a single graph for each observer, the effects of interest are difficult to detect because of the large number of curves. For clarity, the curves for each observer have been plotted in two separate graphs: The graphs on the left show the results for leading-stimulus durations of 10, 40, 80, and 120 msec, and the graphs on the right show the results for leading-stimulus durations of 20, 60, 100, and 140 msec.

input filter. This establishes a practical and conceptual link between the present work and the extensive research done on visual temporal responding under varying intensities of stimulation (Barlow, 1958; Bowen et al., 1974; Kelly, 1971).

Second, there is a close match in Figures 10 and 11 between the slant of the fitted contours (heavy lines) and that of the iso-accuracy contours defined by the transitions between adjacent gray bands. It goes without saying that, as in Experiment 1, predictions from the storage and the processing hypotheses are disconfirmed by the slant of the iso-accuracy contours; the former predicts horizontal contours and the latter predicts contours much steeper than were obtained.

A further aspect of the iso-accuracy contours should be highlighted: The remarkable similarity in slant among all iso-accuracy contours in Experiments 1 and 2 (Figures 4,10 , and 11) suggests that the factor(s) underlying these slants remained invariant across the experimental manipulations. In the following text, we suggest that this invariance represents a fundamental characteristic of the temporal correlation process. For simplicity, let us consider only the $50 \%$ iso-accuracy contours, namely, the contours fitted by the middle heavy line in each panel of 


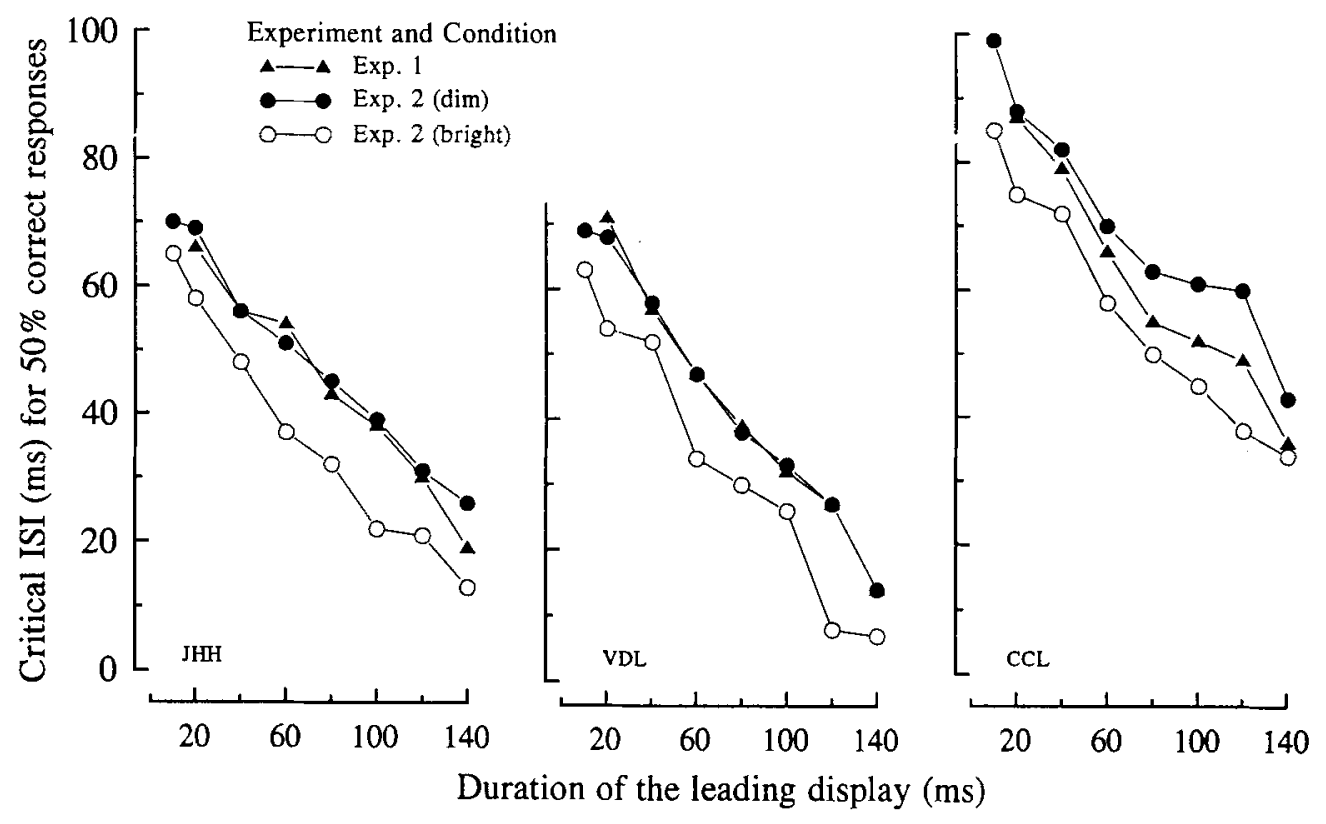

Figure 8. Duration of ISI (obtained by interpolation) that yielded $50 \%$ correct responses at each duration of the leading stimulus in Experiment 1 and in the two viewing conditions of Experiment 2. The results of individual observers are shown in separate panels. An inverse duration effect is evidenced by the negative slopes of the plots. An inverse intensity effect is evidenced by the lower level of the plots for the bright condition in relation to the dim condition.

Figures 4,10 , and 11 . These contours show the critical ISIs required to produce $50 \%$ correct performance at each stimulus duration. That is, the contours can be regarded as representing the functional relationship between an independent variable (stimulus duration) and a dependent variable (critical ISI). In this sense, each contour line can be regarded as representing the regression of critical ISI on stimulus duration.

The relationship between stimulus duration and critical ISI for $50 \%$ correct performance in Experiments 1 and 2 is illustrated in Figure 8 . The plots are essentially parallel; for each observer, the relationship is well approximated by a linear regression with slope approximating -.4 . Individual slopes are reported in Table 4 . We regard the model's ability to account for this relationship as strong support for our proposals. Notably, the relationship does not depend on a particular choice of parameter estimates; virtually identical slopes are found with any plausible set of values. This strongly suggests that the relationship is inherent in the structure of the model and in the principles of linear filtering used to derive the visual responses.

\section{GENERAL DISCUSSION}

\section{Comparison With Other Models}

Throughout this article, the rationale for the empirical work was provided by a juxtaposition of three hypotheses of temporal integration: storage, processing, and tem- poral correlation. Thus far, we have made no reference to a fourth model that has been proposed by Loftus and co-workers. In that model, temporal integration is said to be based on rate of information extraction (e.g., Loftus \& Hanna, 1989). In some respects, the informationextraction model bears similarities to earlier storage models. However, continued visibility of a display after stimulus offset is said to depend not only on the amount of decay during the ISI (measured from stimulus offset), but also on the diminishing rate of information extraction whose decay is said to be time locked to stimulus onset. A more comprehensive description of the informationextraction model has been provided by Loftus et al. (1992). The present experiments were not designed to differentiate the information-extraction model from other models of temporal integration; nevertheless, the model

Table 2

Separate Parameter Estimates for Each Observer in Experiment 2

\begin{tabular}{lrrr}
\hline \multicolumn{1}{c}{ Parameter } & J.H.H. & V.D.L. & C.C.L. \\
\hline$\tau$, bright dots* & 12.9 & 14.0 & 19.4 \\
$\tau$, dim dots* & 15.3 & 17.1 & 24.6 \\
$\quad n$ & 7.13 & 7.25 & 6.12 \\
$r_{c}$ & 0.390 & 0.466 & 0.467 \\
$a$ & 0.984 & 0.942 & 0.982 \\
$g$ & 0.081 & 0.094 & 0.171 \\
Root-mean squared & 0.036 & 0.047 & 0.043 \\
$\quad$ error of prediction & & &
\end{tabular}

*In milliseconds. 
Table 3

Parameter Estimates for Experiments 1 and 2 Combined Separately for Each Observer

\begin{tabular}{lcrr}
\hline \multicolumn{1}{c}{ Parameter } & J.H.H. & V.D.L. & C.C.L. \\
\hline$\tau$ (Experiment 1)* & 16.1 & 16.6 & 21.0 \\
$\tau$, dim dots (Experiment 2)* & 16.0 & 16.0 & 23.6 \\
$\tau$, bright dots (Experiment 2)* & 13.4 & 13.4 & 18.7 \\
$n$ & 7.01 & 6.63 & 5.90 \\
$r_{c}$ & 0.411 & 0.445 & 0.442 \\
$a$ & 0.989 & 0.965 & 0.996 \\
$g$ & 0.081 & 0.079 & 0.146 \\
Root-mean squared error of prediction & 0.034 & 0.046 & 0.040 \\
\hline *In milliseconds.
\end{tabular}

does make explicit predictions regarding the effects of stimulus duration and ISI under the conditions employed in Experiment 1. Therefore, it behooves us to assess the model's fit to the experimental outcome.

We implemented a computer simulation of the information-extraction model, and derived predictions for the results of Experiment 1 . The predictions provided a good fit to the data. Indeed, the fit was virtually indistinguishable from that derived from the temporal correlation model. However, the present work had not been planned as a test of the two models, so the similarity in predic- tions cannot be taken as an indication of unconditional equivalence. It goes without saying that if the two models are to be separated on empirical grounds, experiments must be designed expressly for that purpose.

\section{Locus of Temporal Correlation}

If, as we propose, integration is governed by correlational principles, we might speculate both on the neurophysiological implementation of the correlational process and on the level at which the processing might take place. As noted previously, correlational principles have been
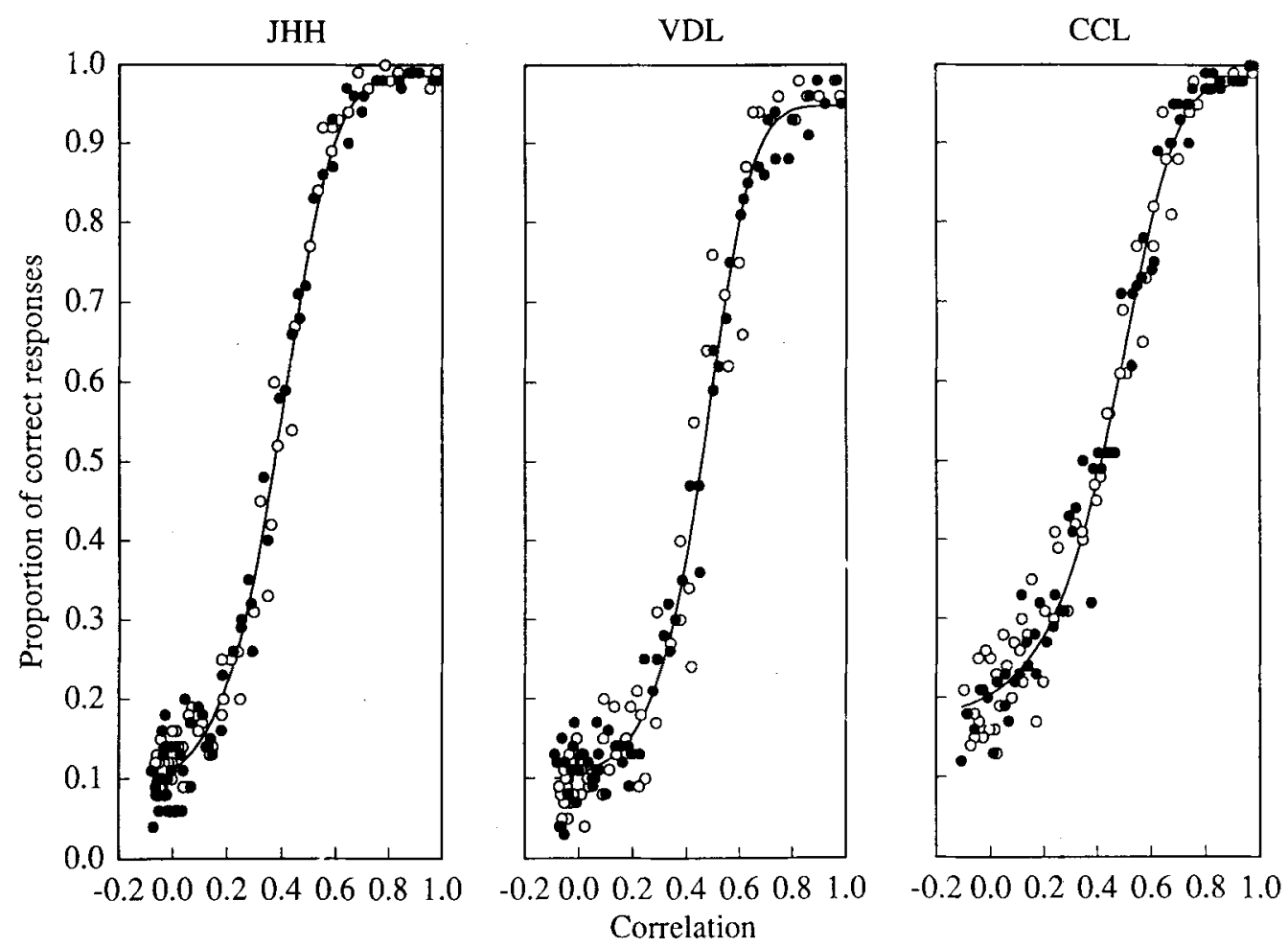

Figure 9. Relationship between accuracy of performance in Experiment 2 and the correlation between visual responses computed on the basis of Equation 10. Within each panel, the continuous curve shows the model's fit to an observer's performance. Individual dots represent the proportion of correct responses obtained by each observer in each condition of Experiment 2. Results with bright and dim stimuli are represented by open and filled symbols, respectively. Details of the parametric fits are given in Table 2. 

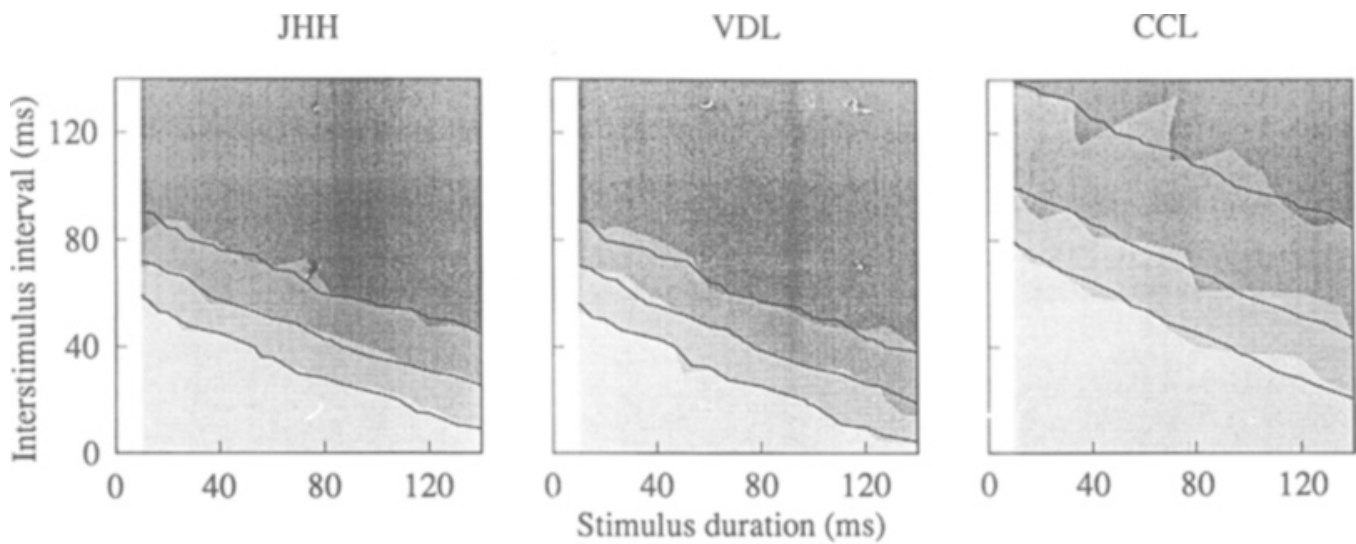

Figure 10. Contour plots of accuracy of performance in the dim viewing condition of Experiment 2 as a function of stimulus duration and interstimulus interval. For details, see caption of Figure 4.

used extensively in theories of spatial vision. In some instances, specific underlying mechanisms have been proposed (cf. Uttal, 1973, 1975). In a similar vein, correlational principles have been invoked in accounts of spatiotemporal events such as motion perception (e.g., Reichardt, 1961) and metacontrast masking (e.g., Bridgeman, 1978).

In general, peripheral correlational mechanisms have met with only limited success in accounting for complex perceptual phenomena. For example, the explanatory scope of the Reichardt motion sensor is restricted by such limitations as aperture problems and a limited range of plausible temporal constants. To account for complex aspects of motion perception, more central mechanisms have been proposed (e.g., Adelson \& Movshon, 1982). We believe that a peripheral correlational mechanism would encounter much the same problems in accounting for temporal integration and segregation.
Strictly peripheral mechanisms are strained in accounting for the extant data on temporal integration. As shown in Figures 2 and 7, integration can occur over temporal intervals that are implausibly long for such mechanisms. Also incompatible with a peripheral locus is the effect of trailing-stimulus duration: the probability of two stimuli being coded as temporally disjoint increases with the duration of the trailing stimulus (Dixon \& Di Lollo, in press). The strength of this effect continues to increase up to trailing-stimulus durations of several hundred milliseconds, an excessively long period for strictly peripheral mechanisms.

Perhaps the most telling argument favoring a central locus is the relative complexity of the hypothesized correlational process involving, as it does, the emergence of a temporal code that determines whether stimuli presented early in a temporal sequence are to be perceived as integrated with-or segregated from-stimuli presented later
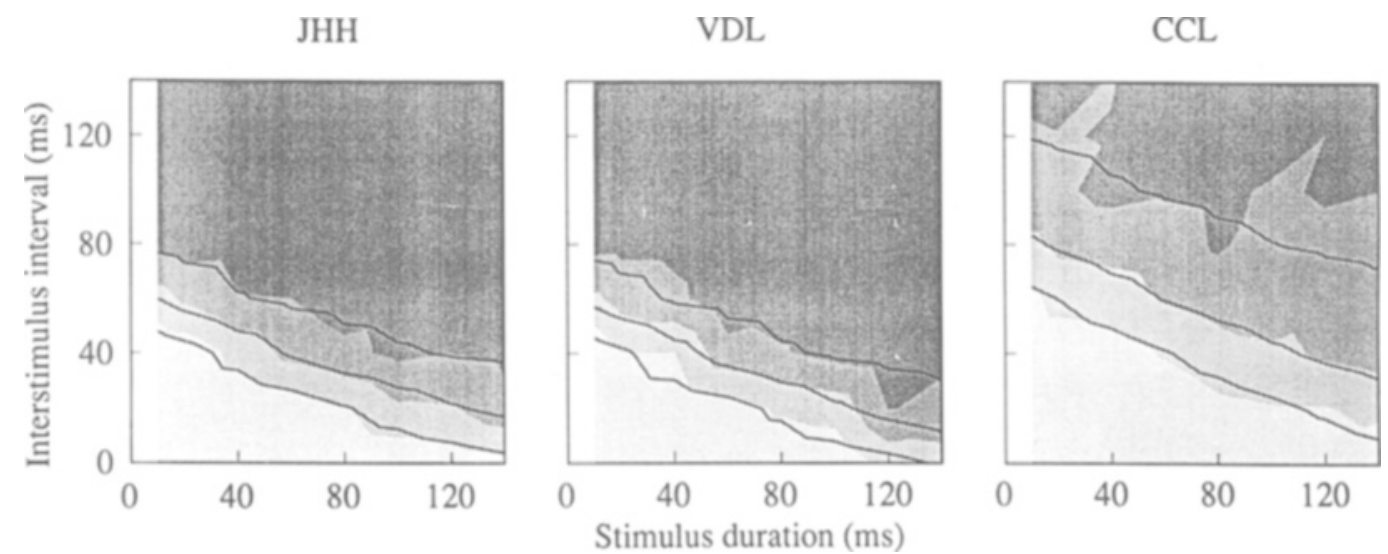

Figure 11. Contour plots of accuracy of performance in the bright viewing condition of Experiment 2 as a function of stimulus duration and interstimulus interval. For details, see caption of Figure 4. 
Table 4

Slopes of Linear Regression of Critical ISI (for 50\% Correct Responses) on Duration of Leading Display in

Experiments 1 and 2, Shown Separately for Each Observer

\begin{tabular}{llll}
\hline \multicolumn{1}{c}{ Experiment/ } & & & \\
\multicolumn{1}{c}{ Condition } & J.H.H. & V.D.L. & C.C.L. \\
\hline Experiment 1 & -0.38 & -0.44 & -0.41 \\
Experiment 2 (dim) & -0.34 & -0.42 & -0.37 \\
Experiment 2 (bright) & -0.39 & -0.44 & -0.39 \\
\hline
\end{tabular}

in the sequence. Local peripheral mechanisms are almost certainly involved in initial stages such as spatiotemporal filtering and, possibly, implementation of the sliding temporal window. However, although fundamental to the final outcome, these processes must be regarded as preliminary events. On these considerations, the conclusion is almost inescapable that the all-important temporal code is the outcome of activity at higher processing levels.

\section{REFERENCES}

Adelson, E. H., \& Movshon, J. A. (1982). Phenomenal coherence of moving visual patterns. Nature, 300, 523-525.

AlLPORT, D. A. (1968). Phenomenal simultaneity and the perceptual moment hypothesis. British Journal of Psychology, 59, 395-406.

BARLOW, H. B. (1958). Temporal and spatial summation in human vision at different background intensities. Journal of Physiology, 141, 337-350.

Bowen, R. W., Pola, J., \& Matin, L. (1974). Visual persistence effects of flash luminance, duration, and energy. Vision Research, 14, 295-303.

Bretrmeyer, B. G., Kropfl, W., \& Julesz, B. (1982). The existence and role of retinotopic and spatiotopic forms of visual persistence. Acta Psychologica, 52, 175-196.

Bridgeman, B. (1978). Distributed sensory coding applied to simulation of iconic storage and metacontrast. Bulletin of Mathematical Biology, 40, 605-623.

Coltheart, M. (1980). Iconic memory and visible persistence. Perception \& Psychophysics, 27, 183-228.

Di LoLLo, V. (1980). Temporal integration in visual memory. Journal of Experimental Psychology: General, 109, 75-97.

Di Lollo, V., \& Dixon, P. (1988). Two forms of persistence in visual information processing. Journal of Experimental Psychology: Human Perception \& Performance, 14, 671-681.

Di LolLo, V., \& FinLEY, G. (1986). Equating the brightness of brief visual stimuli of unequal durations. Behavior Research Methods, Instruments, \& Computers, 18, 582-586.

Dixon, N. F., \& Hammond, E. J. (1972). The attenuation of visual persistence. British Journal of Psychology, 63, 243-254.

Dixon, P., \& Di Lollo, V. (in press). Beyond visible persistence: An alternative account of temporal integration and segregation in visual processing. Cognitive Psychology.

DoDWELL, P. C. (1971). On perceptual clarity. Psychological Review, 78, 275-289.

EFron, R. (1970). Effect of stimulus duration on perceptual onset and offset latencies. Perception \& Psychophysics, 8, 231-234.

EFroN, R., \& LEE, D. N. (1971). The visual persistence of a moving stroboscopically illuminated object. American Journal of Psychology, 84, 365-375.
FINLEY, G. (1985). A high-speed point plotter for vision research. Vision Research, 25, 1993-1997.

FISHER, R. A. (1922). The goodness of fit and regression formulae, and the distribution of regression coefficients. Journal of the Royal Statistical Society, 85, 597-612.

GLASS, L. (1969). Moiré effect from random dots. Nature, 223, 578-580.

Graham, N., Hood, D. C. (1992). Modeling the dynamics of light adaptation: The merging of two traditions. Vision Research, 32, 1373-1393.

HABER, R. N., \& Standing, L. (1969). Direct measures of short-term visual storage. Quarterly Journal of Experimental Psychology, 21, 43-54.

Hogben, J. H., \& Di Lollo, V. (1974). Perceptual integration and perceptual segregation of brief visual stimuli. Vision Research, 14, 1059-1069.

Hogben, J. H., \& Di Lollo, V. (1985). Suppression of visible persistence in apparent motion. Perception \& Psychophysics, 38, 450-460.

Hood, D. C., \& Grover, B. G. (1974). Temporal summation of light by a vertebrate visual receptor. Science, 184, 1003-1005.

IRWIN, D. E., \& Yeomans, J. M. (1986). Sensory registration and informational persistence. Journal of Experimental Psychology: Human Perception \& Performance, 12, 343-360.

IvEs, H. E. (1922). A theory of intermittent vision. Journal of the Optical Society of America, 6, 343-361.

KeLLY, D. H. (1961). Visual responses to time-dependent stimuli: II. Single-channel model of the photopic visual system. Journal of the Optical Society of America, 51, 747-754.

KeLLY, D. H. (1971). Theory of flicker and transient responses: 1. Uniform fields. Journal of the Optical Society of America, 61, 537-546.

Loftus, G. R., Duncan, J., \& Gehrig, P. (1992). The time course of perceptual information that results from a brief visual presentation. Journal of Experimental Psychology: Human Perception \& Performance, 18, 530-549.

Loftus, G. R., \& HaNNA, A. M. (1989). The phenomenology of spatial integration: Data and models. Cognitive Psychology, 21, 363-397.

Neisser, U. (1967). Cognitive psychology. New York: AppletonCentury-Crofts.

REICHARDT, W. (1961). Autocorrelation, a principle for the evaluation of sensory information. In W. A. Rosenblith (Ed.), Sensory communication (pp. 303-317). Cambridge, MA: MIT Press.

RouFs, J. A. J. (1972a). Dynamic properties of vision: I. Experimental relationships between flicker and flash thresholds. Vision Research, 12, 261-278.

Rours, J. A. J. (1972b). Dynamic properties of vision: II. Theoretical relationships between flicker and flash thresholds. Vision Research, 12, 279-292.

Shiorri, S., \& Cavanagh, P. (1992). Visual persistence of figures defined by relative motion. Vision Research, 32, 943-951.

SPERLING, G. (1971). The description and luminous calibration of cathode ray oscilloscope visual displays. Behavior Research Methods \& Instrumentation, 3, 148-151.

UENO, T. (1977). Reaction time as a measure of temporal summation at suprathreshold levels. Vision Research, 17, 227-232.

UtTaL, W. R. (1973). The psychobiology of sensory coding. New York: Harper \& Row.

UTTAL, W. R. (1975). An autocorrelation theory of form detection. Hillsdale, NJ: Erlbaum.

WAtSon, A. B. (1986). Temporal sensitivity. In K. R. Boff, L. Kaufman, \& J. P. Thomas (Eds.), Handbook of perception and human performance (pp. 6-1-6-43), New York: Wiley.

(Manuscript received April 1, 1993; revision accepted for publication September 2, 1993.) 